\title{
L'enfant prend la parole : un tournant récent de notre histoire catéchétique
}

\section{Raymond Anctil}

Volume 46, 1979

URI : https://id.erudit.org/iderudit/1007113ar

DOI : https://doi.org/10.7202/1007113ar

Aller au sommaire du numéro

Éditeur(s)

Les Éditions Historia Ecclesiæ Catholicæ Canadensis Inc.

ISSN

0318-6172 (imprimé)

1927-7067 (numérique)

Découvrir la revue

Citer cet article

Anctil, R. (1979). L'enfant prend la parole : un tournant récent de notre histoire catéchétique. Sessions d'étude - Société canadienne d'histoire de l'Église catholique, 46, 25-44. https://doi.org/10.7202/1007113ar

Tous droits réservés @ Les Éditions Historia Ecclesiæ Catholicæ Canadensis Inc., 1979
Ce document est protégé par la loi sur le droit d'auteur. L’utilisation des services d'Érudit (y compris la reproduction) est assujettie à sa politique d'utilisation que vous pouvez consulter en ligne.

https://apropos.erudit.org/fr/usagers/politique-dutilisation/ 


\section{L'enfant prend la parole : un tournant récent de notre histoire catéchétique}

Il est difficile, du moins dans le contexte québécois, de parler de l'enfant sans aborder la question de son éducation et de sa formation religieuse en milieu scolaire. Ce souci d'éducation et cette préoccupation de la foi répondent d'ailleurs à la sollicitude de l'Église envers l'enfant et envers les droits de l'enfant, tout particulièrement en cette année consacrée à l'enfance. Comme l'écrivait récemment le pape Jean-Paul II :

Dans l'éducation à laquelle contribuent, avec les parents, l'école et d'autres organismes de la société, l'enfant doit trouver les possibilités "de se développer d'une façon saine et normale sur le plan physique, intellectuel, moral, spirituel et social, dans ces conditions de liberté et de dignité ", comme l'affirme le deuxième principe de la Déclaration des droits de l'enfant. À ce sujet, l'enfant a droit également à la vérité, dans un enseignement qui tienne compte des valeurs éthiques fondamentales, et qui rend possible une éducation spirituelle. conformément à l'appartenance religicuse de l'enfant, à l'orientation voulue légitimement par ses parents et aux exigences d'une liberté de conscience bien comprise, à laquelle le jeune doit être préparé et formé tout au long de l'enfance et de l'adolescence. Sur ce point, il est normal que l'Église puisse faire valoir ses propres responsabilités !.

La présente communication voudrait s'intéresser tout particulièrement à un tournant récent de notre histoire catéchétique, c'est-à-dire à ce que nous pourrions appeler la «prise de parole » de l'enfant dans la catéchèse québécoise de ces dernières années.

1 Jean-Paul II, «I Ies droits de l'enfant», dans Documentation catholique, 61" année, t. LXXVI, n" 1757, 4 février 1979, p. 102. 


\section{BRÈVE HISTOIRE DE LA CATÉCHÈSE QUÉBÉCOISE}

Une brève histoire des catéchismes au Québec s'avère très utile : elle circonscrit la situation présente, elle en vérifie l'authenticité et elle devient source de promesse. En effet, la réalité présente est d'autant plus enrichissante qu'elle révèle les sources d'où elle jaillit, qu'elle construit son avenir dans la fidélité qui se fonde sur la continuité, qu'elle prolonge. renouvelle et transforme le germe qui l'a vu naître ${ }^{2}$.

Diverses approches peuvent mettre en relief les orientations de la catéchèse. Il est possible en effet de côtoyer les grands événements de l'histoire profane ou encore de suivre les grandes étapes de l'éducation au Québec. Il serait encore possible de privilégier un auteur, comme le fait Fernand Porter ${ }^{3}$ ou Benoit Boily ${ }^{4}$. ou un thème, à la manière d'Étienne Fortier ${ }^{5}$. Sans nier le bien fondé d'une approche événementielle, chronologique ou thématique de l'histoire, il apparaît plus utile pour ce propos de faire appel à une approche des mentalités.

Dans cette perspective, trois grands mouvements ont marqué l'histoire catéchétique du Québec: le mouvement institutionnel qui met en place les formes et les structures utiles à un bon encadrement de la foi, le mouvement méthodologique qui apporte de lintérieur même de la catéchèse des méthodes nouvelles et une meilleure adaptation de la catéchèse aux personnes, enfin un troisième mouvement s'attaque aux orientations fondamentales de la catéchèse en notre milieu.

\section{LES PIONNIERS}

Le premier mouvement voit progressivement surgir selon les circonstances et les événements, tant profanes que religieux. des

2 François Coudreau. «L'enracinement dans le passé de la vie actuelle du croyant », dans Catéchèse. t. 10. n” 39, avril 1970, pp. 151-170.

3 Fernand Porter, o.f.m., L'institution catéchistique au Canada. Deux siècles de formation religieuse. 1633-1833, Montréal. Les Éditions Franciscaines, 1949.

4 Benoît BoIly, Le catéchisme du diocèse de Québec, 1702. Son auteur, ses sources, son contenu. Thèse dactylographiée présentée à la faculté de théologie de Paris, le 5 mai 1966.

5 Étienne FOrTIER, La Tradition catéchétique canadicnnc. Thèse dactylographiée présentée à l'Institut Catholique de Paris. t. 1 : la foi ; t. $2: 1$ l'Église. Paris, mai 1964. 
institutions qui donnent au catéchisme canadien un caractère particulier. Il naît en terre d'Amérique avec le Catéchisme de $M^{g r}$ de Saint-Vallier, il se poursuit avec les multiples adaptations du Catéchisme de Sens, il s'achève avec la traduction du Catéchisme américain dit de Baltimore. Cependant, cette tradition catéchétique à forme institutionnelle s'enracine dans un grand moment de l'histoire de l'Église catholique : le Concile de Trente.

Ce Concile s'inscrit dans le courant de la Renaissance et de la Réforme protestante. La Renaissance tente un «aggiornamento» culturel par le retour aux valeurs de la culture antique; elle influence l'éducation au point que les Rabelais (1483-1553), les Montaigne (1533-1592), inspirés par une profonde connaissance de l'homme, s'opposent à une éducation livresque, pédante et sévère et suggèrent l'utilisation de disciplines pratiques et utiles ainsi que l'usage de méthodes actives dans le but de rendre l'homme capable de s'adapter à la vie de son temps.

En cette même période, de nombreuses difficultés agitent l'Église. Martin Luther (1483-1546) déclenche un mouvement majeur de contestation dans l'Église. Sa critique sévère des dogmes, son intérêt particulier pour l'individu et son interprétation libre de l'Écriture constituent autant d'éléments réformateurs. Pour assurer le succès de sa révolution religieuse, Luther mise sur l'éducation. Il conseille à tous les seigneurs et magistrats de prendre en main l'instruction et l'éducation des enfants. Il propose comme principes d'éducation de remettre en valeur la responsabilité des parents en ce domaine, de rendre l'école obligatoire et de bien choisir les maîtres de l'enseignement religieux. Il incite les citoyens à ériger des écoles chrétiennes dans le but de former des laïcs responsables du Royaume et, éventuellement, de former de futurs prêtres.

L'Église catholique ne peut rester indifférente aux mutations culturelles ambiantes. Provoquée par les récentes transformations éducatives et religieuses, elle se voit dans l'obligation de repenser sa mission prophétique et ses moyens d'évangélisation. Dans la continuité des conciles provinciaux de Rouen en 1445 et de Narbonne en 1541, qui insistent pour faire revivre les écoles de toutes sortes (cathédrales, paroissiales, monastiques), l'Église du Concile de Trente entreprend une réforme en son propre sein et attache une grande importance à l'éducation. Dans cette intention, le Concile décrète le droit des enfants à l'éducation, l'établissement des écoles populaires, la nomination des maîtres par les évêques; autant de 
dispositions qui démontrent une Église préoccupée par les problèmes de l'enseignement et de l'éducation.

Le Concile de Trente veut donc répondre à l'ampleur de la prédication protestante. Il charge quelques-uns de ses membres de rédiger un catéchisme. Destiné surtout aux prédicateurs, le catéchisme paraît en 1566. Malgré ses nombreuses qualités, ce catéchisme ne réussit pas à s'imposer comme catéchisme universel ${ }^{6}$. Cependant, peu après le Concile, naît l'institution catéchétique pour les enfants. Les évêques, soucieux d'enseigner comme le recommandait le Concile, partagent cette responsabilité non seulement avec les curés et les parents mais aussi avec les maîtres et les personnes intéressées à cette fonction ecclésiale. D'où la fondation d'ordres et de confréries pour l'instruction religieuse des enfants ${ }^{7}$. Il en résulte une multiplication de catéchismes. Chaque évêque désire son propre catéchisme diocésain. Ces catéchismes sont d'abord rédigés pour répondre aux besoins de la formation religieuse des adultes et des adolescents. Certains catéchismes préparent même à la première communion et à la confirmation.

De tous les catéchismes de cette période, il faut particulièrement noter un catéchisme d'avant Concile, celui de Canisius (1524-1597) qui s'inspire fortement de l'Écriture et des Pères, le Catéchisme du Concile lui-même (1566) qui reflète les influences du milieu socioculturel et un catéchisme d'après le Concile, le catéchisme de Bellarmin (1542-1621) qui dénote une théologie de controverse, et enfin, un peu plus tard, paraîtront les catéchismes de Bossuet qui se situent à mi-chemin entre la mentalité du Concile de Trente et la mentalité de Bellarmin ${ }^{8}$.

Cette période plutôt marquée par une structure théologique à caractère apologétique s'éloigne progressivement des sources bibliques et liturgiques; elle développe des attitudes de respect envers la tradition qui revêtent une couleur fixiste et conservatrice. C'est dans ce climat que naît le premier catéchisme québécois.

6 Norbert Fournier, Exigences actuclles de la Catéchèse; essai de synthèse en pédagogie de la religion. Montréal, Les Clercs de Saint-Viateur, 1960 , p. 26.

7 Joseph Colomв, Le Service de l'Évangile. Manuel catéchétique, t. 1, Paris, Desclée et Cie, 1968, p. 39.

8 Joseph СоLомв, op. cit., p. 41 , note 4. 
Avant le catéchisme de $\mathbf{M}^{* *}$ de Saint-Vallier «il n'existe pas de catéchisme officiel ${ }^{9}$ ». Les catéchismes qui circulent alors en Nouvelle-France sont les «différents catéchismes diocésains des villages et campagnes de France d'où venaient les premiers habitants et les prêtres 10 ». Ces catéchismes étaient surtout ceux des diocèses «de Paris, de Rouen et celui (catéchisme) des Jésuites 11 ». Il est fort possible que les catéchismes utilisés par les Jésuites du Québec soient ceux de Canisius et de Bellarmin.

La rareté et la diversité des manuels de religion en usage dans son diocèse ${ }^{12}$, son caractère énergique, son devoir de pasteur et la conscience de sa mission incitent $\mathbf{M}^{\mathrm{*r}}$ de Saint-Vallier à écrire son propre catéchisme diocésain, comme les évêques français, afin de mieux aider les parents à faire apprendre à leurs enfants les rudiments de la foi et les principes de la vie chrétienne.

Après avoir parcouru son diocèse pendant cinq mois pour bien connaître les besoins de ses diocésains ${ }^{13}$ et après avoir travaillé de nombreuses années à la rédaction de son catéchisme sous l'influence «des figures les plus attachantes de la catéchétique française (Bossuet, Fleury, Fénélon) », $\mathbf{M}^{\mathrm{*r}}$ de Saint-Vallier fait paraître en 1702 le «seul catéchisme qui fut jamais totalement conçu pour le Canada ${ }^{14}{ }$.

Ce catéchisme n'a pratiquement vécu que 50 ans ; son influence directe ne dépasse pas beaucoup la conquête anglaise. Toutefois, le visage austère de la vie chrétienne qu'il préconise s'est transmis jusqu'à nos jours 15 et marque ainsi "pour toujours la mentalité de l'éducation religieuse élémentaire pour plus de deux siècles ${ }^{16}{ }^{»}$.

Avec l'écoulement de l'unique édition du Catéchisme de $M^{a r}$ de Saint-Vallier ${ }^{17}, \mathrm{M}^{\mathrm{rr}}$ de Pontbriand en 1754 adopte le Catéchisme

9 Étienne Fortier, op. cit., t. 1, p. VIII.

10 Marcel Caron, "Quelques pages d'histoire », dans Le Souffle, no 32, juin 1970, p. 2.

$11 \mathrm{M} *$ r Joseph-Arthur PAPINEAu, Le prêtre et l'enseignement catéchistique, Joliette, Office catéchistique diocésain, 1954, pp. 13 et 14.

12 Guy Plante, Le rigorisme all XVII' siècle. M!r de Saint-Vallier et le saciement de pénitence (1685 et 1727), Gembloux, J. Duculot, 1971, p. 45.

13 Guy Plante, op. cit., pp. 33 et 52.

14 Étienne FortIER, op. cit., t. 1, p. VIII.

15 Guy Plante, op. cit., p. 171.

16 Fernand PORTER, op. cit., p. 164.

17 Fernand PORTER, op. cit., p. 117. 
de $M^{\prime \prime r}$ Languet nommé archevêque de Sens en 1730. Ce catéchisme qui circulait déjà subrepticement depuis un certain temps est reconnu officiellement par $\mathrm{M}^{* r}$ Briand en 1777. Ce catéchisme sera réédité 10 fois en moins de 50 ans ${ }^{18}$.

Le Catéchisme de Sens était composé d'un Grand Catéchisme et d'un Petit Catéchisme. Le Grand Catéchisme fut, dès sa reconnaissance officielle, l'objet de transformation. $\mathbf{M}^{\mathrm{gr}}$ Briand et $\mathbf{M}^{\mathrm{sr}}$ Mongolfier tentent d'en améliorer la pédagogie afin de faciliter la connaissance et la mémorisation.

M*r Plessis, quelques années plus tard, trouve que ces grands savants n'ont pas atteint leurs objectifs. Pour ne pas tomber dans le piège de ses prédécesseurs, $M^{*}$ Plessis ouvre une large consultation auprès de ceux qui exercent cette fonction de catéchiste afin que le catéchisme soit l'œuvre de praticiens. Cette consultation des catéchistes à tous les niveaux de responsabilité aboutit à la production d'un nouveau Petit Catéchisme, reconnu officiellement le $1^{\text {er }}$ avril 1815 .

$\mathrm{M}^{* *}$ Bernard-Claude Pañet succède à $\mathrm{M}^{\mathrm{*}}{ }^{*}$ Plessis. Il souscrit entièrement aux réformes du Petit Catéchisme de son prédécesseur. Dans la nécessité de réimprimer le Grand Catéchisme de Sens, il profite de l'occasion pour opérer quelques retouches mineures à ce catéchisme ${ }^{19}$.

Un peu plus tard, le Premier Concile Provincial des évêques du Québec veut "poser la base de l'uniformité dans l'enseignement religieux en adoptant un catéchisme commun à tous les diocèses ${ }^{20}$ ». Tout en maintenant en substance le Petit Catéchisme et le Grand Catéchisme déjà utilisés, ce concile augmente le contenu du Petit Catéchisme en vue d'établir une plus grande cohérence avec le Grand Catéchisme. Il répartit la matière selon la disposition du catéchisme du Concile de Trente et s'efforce d'en améliorer la pédagogie.

Avec beaucoup de soin et de chaleur les évêques insistent sur l'obligation qu'ont les pasteurs et les parents de veiller à l'éducation

18 Ibid., p. 117.

19 M $\%$ B Bernard-Claude Panet, «Mandement au sujet d'une nouvelle édition du Grand Catéchisme », dans $M E Q$, III, pp. 233-234.

20) Mrr Pierre-Flavien Turgeon, "Mandement de Monseigneur l'Archevêque de Québec pour la publication du petit catéchisme rédigé par l'ordre du premier concile provincial », dans $M E Q, I V$, p. 91. 
chrétienne des enfants. Dépositaires de la science sacrée, les prêtres doivent enseigner le catéchisme tous les dimanches de l'année dans un langage simple ${ }^{21}$. La collaboration des parents est aussi nécessaire pour enseigner les premières vérités de la religion aux enfants. En leur faisant poser des actes de foi, d'espérance et de charité, en les conduisant régulièrement au catéchisme du dimanche, en leur donnant le bon exemple, les parents collaborent à une meilleure éducation chrétienne de leurs enfants.

L'amélioration du Catéchisme de Sens par le Premier Concile Provincial de Québec n'évite pas la critique. Une consultation est à nouveau lancée auprès des catéchistes pour savoir s'il ne faut pas le remplacer par un autre ou le modifier.

De leur côté, les évêques américains, motivés par les assauts du libéralisme, par le désir d'une plus grande uniformité et d'une plus grande discipline ainsi que par les visées d'intégrité et de centralisme de Vatican I, tentent d'élaborer un catéchisme unique. Leurs efforts aboutissent au Catéchisme de Baltimore. Voici brièvement les antécédents de ce catéchisme selon Charles J. Carmody ${ }^{22}$.

Après les efforts de Vaux pour adapter le catéchisme du Concile de Trente, apparaît un nouveau Catéchisme intitulé An Abridgement of Christian Doctrine généralement attribué à Henry Tuberville. Ce catéchisme subit l'influence évidente de Canisius et de Bellarmin. Cependant, Tuberville fait œuvre originale avec son catéchisme : il le fait plus adapté à son pays et il répond davantage aux aspirations de son temps. Richard Challoner reprend et révise ce catéchisme de Tuberville qui a déjà subi de nombreuses transformations, demeurant cependant toujours essentiellement le même. James Butler II, qui subit l'influence des catéchismes de Fleury et de Doway, publie son catéchisme en Irlande vers 1775 . Toutes ces sources avec le Carroll Catechism ont alimenté les intentions des évêques du Concile Provincial de Baltimore en 1829, du premier et second Concile Plénier de Baltimore en 1852 et 1866 , du troisième concile plénier de 1884-1885 où un comité consultatif étudie la question d'un

21 M ${ }^{*}$ Pierre-Flavien Turgeon et Évêques de LA Province EccléSIASTIQue DE QuÉBEC, «Mandement de l'Archevêque et des Évêques de la Province ecclésiastique de Québec promulgant le nouveau catéchisme, rédigé par l'ordre du premier concile provincial », dans $M E Q$, IV, pp. 99-100.

22 Charles J. CARMODY, The Roman Catholic Catechesis in the United States 1784-1930: A Study of its Theory, Development and Materials, Loyola University of Chicago, 1975. 
catéchisme unique. C'est ainsi qu'apparaît en 1885 le Catéchisme de Baltimore qui, après augmentation, est approuvé par l'Archevêque et les Évêques des Provinces Ecclésiastiques de Québec le 20 juin 1888 ; il est prescrit par le Cardinal Elzéar-Alexandre Taschereau le 15 mai $1894^{23}$.

Les nombreux manuels en usage au niveau du secondaire privé sont surtout ceux de Scheffmacker, de Gaume et de Schouppe. Joseph Hallé, au congrès de l'enseignement secondaire tenu à Québec les 20-21 juin 1914, souligne que Cauly et Duvivicr ont tendance à remplacer Gaume ${ }^{24}$. Ce catéchisme de Cauly est déjà rendu à sa $56^{\prime \prime}$ édition en 1915. Il commente le catéchisme des provinces et influence, avec le catéchisme expliqué de son contemporain Lasfargues, la mentalité religieuse des Québécois durant des années ${ }^{25}$.

\section{LES PRÉCURSEURS}

Trois grands catéchismes ont marqué jusqu'ici la tradition catéchétique québécoise. Ces catéchismes de $\mathbf{M}^{*}$ de Saint-Vallier, de Sens et de Baltimore ont rempli unc remarquable fonction dans la formation spirituelle du peuple québécois. L'histoire de ces catéchismes dénote le souci constant apporté par les évêques, les curés et les professeurs à l'enseignement religieux des adultes et des enfants.

Cependant, tous ces efforts ont porté plutôt sur ce qui doit être transmis au chrétien pour qu'il puisse faire son salut que sur le comment le catéchisé peut mieux vivre sa foi, c'est-à-dire les efforts ont porté plus sur le contenu de l'enseignement religieux que sur la pédagogie catéchétique elle-même. Au début du $\mathrm{XX}^{\circ}$ siècle, toutefois, cette question pédagogique, sous-jacente dans la tradition catéchistique, se pose de plus en plus lucidement. Elle provoque une recherche intensive sur les méthodes en enseignement religieux.

23 Luche Amans, Notes d'un catéchiste ou Court commentairc littéral sur le Catéchisme des provinces ecclésiastiques de Québec, Montréal, Ottawa, par un prêtre du diocèse de Montréal, Montréal, Cadieux et Derome, 1897, p. 3 .

24 Joseph Hallé, «La formation religieuse par l'enseignement », dans Congrès de l'enseignement secondaire, Québec, 1914, Actes du congrès de l'enseignement secondaire, Québec, l'Action sociale, 1915, p. 32.

25 Gabriel Dussault, «La religion de l'ordre ... et après? - Aperçus sur la morale québécoise de $1900 »$, dans Relation, $\mathrm{n}^{\circ}$ 377, décembre 1972, p. 330 . 
Cette période de l'histoire de la catéchèse couvre la première moitié $\mathrm{du} \mathrm{XX}^{\mathrm{e}}$ siècle. Elle prépare un renouveau.

Depuis la conquête anglaise, l'Église catholique se maintient grâce à une forte structure paroissiale et familiale que l'enseignement religieux dans les écoles vient appuyer. Intimement liée à la tradition française, la religion catholique développe un certain antagonisme envers la religion protestante ${ }^{26}$. Cette foi, fondée sur une apologétique défensive, risque de se privatiser et d'éteindre tout dynamisme missionnaire ${ }^{27}$. Cette attitude se reflète dans les manuels didactiques : l'enseignement frise l'apologétique, il s'éloigne de la vie concrète, il préconise une méthode plutôt passive; l'annonce du message vise d'abord la communication d'un savoir et l'instrument tout indiqué pour cette activité pastorale est le catéchisme.

La méthode jusqu'ici utilisée s'inspire fortement de la méthode dite de Saint-Sulpice en raison de la description des méthodes pédagogiques qui en 1915 circulent dans les séminaires. L'insistance sur un apprentissage cognitif de vérité, le maintien d'une discipline rigoureuse et l'importance attribuée à la psychologie relative aux groupes nombreux dénotent, en effet, beaucoup de parenté avec la méthode de Saint-Sulpice. Ces orientations influendent l'enseignement religieux jusque vers 1930 .

$\mathrm{M}^{*}$ Ross, évêque de Gaspé, est un des premiers au Québec à s'élever pour critiquer vertement un enseignement religieux trop théologique, livresque et mécanique. Pour lui, il faut d'abord faire comprendre avant de faire apprendre, il faut s'adresser autant au cœur qu'à l'intelligence, il est nécessaire d'utiliser un langage compréhensible à l'auditoire et de tenir compte des âges et du milicu. C'est proposer en réalité une méthode plus inductive.

Cette dernière méthode semble avoir été introduite au Canada par une brochure publiéc à Ottawa par l'abbé J.J. Gorman en 1931 et par un inspecteur d'école de la Province d'Ontario, John Martin Bennett ${ }^{28}$. Elle est propagée au Québec par M"r F.X. Ross ${ }^{29}$.

26 Pierre R^NwI:7, "Le mouvement catéchétique au Canada français », dans Lumen Vituc, v. 19, n" 4, octobre-décembre 1964, p. 746.

27 Guy de Brfagne. Pastorale catéchétique, Paris, DDB, 1953, p. 184.

28 John Martin BrNne:Tt, Manual of Suggestions in Catechetics, Toronto, Catholic Church Society of Canada, 1934.

29 M"r F.X. Ross, Faut-il un nouve'un catéchisme? Quelle méthode?, Québec, Charrier et Dugal, 1933. 
par C.E. Roy ${ }^{30}$ et plusieurs autres dont M. l'abbé Irénée Lussier, le Père Alcantara Dion, $\mathrm{S}^{\mathrm{r}}$ Saint-Ladislas ${ }^{31}$. Selon l'abbé CharlesEugène Roy dans sa thèse de doctorat en théologie, qui étudie les fondements philosophiques et historiques des méthodes inductives et déductives dans l'enseignement religieux, cette méthode inductive s'inspire des meilleures sources de la tradition catéchétique (Saint François de Sales, Saint Jean-Baptiste de la Salle, Fénélon, Saint Augustin); elle repose sur le principe de l'induction où la nature sensible, le concret et le particulier jouent un grand rôle. Cette méthode inductive intégrale fait donc appel aux sources bibliques. liturgiques, mais aussi aux principes psychologiques et au milieu.

Sœur Saint-Ladislas applique d'une façon remarquable cette méthode active intégrale dans sa collection Aux petits du Royaume vers 1940 qui s'accompagne d'un livre du maître: Catéchisme et Vie chrétienne. Dans ses manuels, elle centre le contenu sur l'incorporation du chrétien au Christ et elle utilise une méthode inductive et intégrale, synthétique et active.

Le Comité Catholique du Conseil de l'Instruction Publique demande le 17 décembre 1942 à sa Commission des Programmes et des Manuels de pourvoir à la révision, à la refonte et à l'amélioration du programme d'études religieuses des écoles élémentaires. Cette commission, après un travail assidu avec des spécialistes, livre ses premiers résultats en juin 1946. Le 7 mai 1948, le Comité Catholique en émet la première édition ${ }^{32}$. Le 15 août 1951, l'édition canadienne du Catéchisme Catholique est approuvée et adoptée dans les classes de troisième à septième années par les Archevêques et Évêques de la province civile de Québec. Ce nouveau programme au niveau élémentaire s'accompagne au niveau secondaire de la collection Témoins du Christ.

Ce Catéchisme Catholique marque une étape et un tournant dans la catéchèse québécoise. Les éditions de 1951 et de 1959 démontrent de nouvelles orientations doctrinales et pédagogiques.

30 C.E. Roy, Méthode pédagogique de l'onscigneme'nt du catéchisme: les fondements philosophiques et historiques, Paris, Tournai, Casterman. 1935.

31 Sour Saint-Ladislas, Alux petits du Royaume: essai pratique de méthodologie catéchistiquc d'après les formules du Catéchisme des provinces ecclésiastiques de Quéhec, Montréal at Ottaw'a. Trois-Rivières, Imprimerie du Bien public, 1941-43.

32 Joseph-Arthur Papineau, Le prêtre d't l'enseigncment catéchistique. Joliette, Office catéchistique diocésain, 1954, pp. 15-16. 
Ce catéchisme devient l'instrument de base pour la formation religieuse des jeunes pour plus d'une décennie ; il suscite des commentaires très élogieux ${ }^{33}$, mais aussi des critiques assez sévères ${ }^{34}$. Les mutations socio-culturelles rapides et irréversibles remettent bientôt en question cet effort pédagogique en enseignement religieux ; elles exigent une réflexion sur les orientations fondamentales de la catéchèse, car un homme nouveau est en train de naître au Québec.

\section{LES RÉALISATEURS}

Conscients de la mutation culturelle du milieu québécois et de la précarité de l'enseignement religieux dans ce nouveau contexte, les évêques créent en 1952 un organisme de consultation et d'exécution en matière catéchétique. Cet organisme, en plus de maintenir des liens étroits avec l'épiscopat en vue de renseigner les évêques sur la situation de l'enseignement religieux, travaille avec les représentants des diverses universités à la coordination des groupes et des comités responsables de la catéchèse ${ }^{35}$. À cet Office Catéchistique Provincial, divers offices diacésains se joignent dont celui de $M^{*}$ J.A. Papineau, inauguré en 1945 selon un décret de la sacrée congrégation du Concile sous Pie XI le 12 janvier 1935. Avec les publications de l'Office Catéchistique Provincial, la circulation des revues européennes de catéchèse au Québec, la formation de nombreux catéchètes canadiens dans les Instituts européens de catéchèse, la naissance de certains Instituts de formation à l'enseignement religieux au Québec, les congrès provinciaux de spécialistes de notre milieu, etc., font que, peu à peu, s'élabore une réflexion proprement canadienne-française en catéchèse. En mai 1962, la Commission épiscopale d'enseignement religieux décide de procéder au renouvellement des manuels de catéchèse de l'élémentaire ${ }^{36}$.

Un comité est aussitôt formé à cet effet. Composée de Réginald Marsolais, Marcel Caron, Jean-Paul Bérubé, cette équipe évalue les manuels en usage au Québec et en Europe, consulte des spécialistes

33 Armand Croteau, "Un nouveau catéchisme canadien », dans Lumen Vitale, v. II, n" 2, avril-juin 1956, p. 395.

34 Jean-Paul AudET, Notre catéchèse est-elle entréc dans une impasse?, Montréal. Institut Dominicain de Pastorale, 1963 (Cahiers de communauté chrétienne).

35 Robert Gaudet, "L'Office Catéchistique Provincial », dans Commulnauté chrétienne, t. 1, n" 5, septembre-octobre 1962, pp. 346-347.

36 Marcel CARON, «Quelques pages d'histoire», dans Le Souffle, n 32, juin 1970, p. 4. 
de renommée mondiale en catéchèse et fait appel à des chercheurs québécois en sciences humaines. Après une année d'expérimentation (1963-1964), paraît en 1964 la première édition de cet «ensemble remarquablement équilibré et complet ${ }^{37}$ »: Viens vers le Père.

La parution de ce premier volume laisse déjà entrevoir les orientations fondamentales du renouveau catéchétique au Québec. Les premières sources de ces orientations sont à chercher dans quatre textes de base: 1) la lettre circulaire des Évêques de la Province de Québec sur "Le Prêtre et l'Enseignement du Catéchisme»; 2) les conclusions du Congrès international de la Catéchèse missionnaire, tenue en Allemagne à Eichstatt du 21 au 28 juillet $1960 ; 3$ ) le commentaire du Programme de Religion des Écoles Élémentaires de la Province de Québec ; 4) le commentaire méthodologique des manuels Témoins du Christ au cours secondaire ${ }^{38}$.

Tous ces textes se concentrent sur quelques éléments essentiels de toute catéchèse : 1) l'école comme lieu de formation: 2) l'importance, la nature et les objectifs de l'enseignement religieux: 3 ) les principales lois de la pédagogie religieuse (adaptationinduction-activité) ; 4) le rôle et les qualités du maître de religion ; 5) l'atmosphère de la classe de religion ${ }^{39}$.

Ce qui est remarquable dans ces principes et dans ces premiers instruments pédagogiques c'est l'originalité de l'œuvre. En effet, le contenu manifeste un effort de synthèse des éléments bibliques, liturgiques, doctrinaux et moraux ; la pédagogie emprunte beaucoup d'éléments à la dynamique de groupe. Ces prises de position révèlent une double inspiration: une inspiration européenne qui fait porter ses efforts sur l'objet même de l'enseignement religieux ${ }^{40}$, considéré à ce moment-là sous son aspect kérygmatique et une inspiration américaine qui fait porter sa recherche en pédagogie sur la psychologie et la sociologie. Le défi consiste précisément à marier ces deux inspirations dans des instruments catéchétiques pour un milieu québécois.

37 Pierre RANwEZ, "Le mouvement catéchétique au Canada français ", dans Lumen Vitcle, v. 19, n* 4, octobre-décembre 1964, p. 751.

38 Office Catéchistique Provincial, Formation du prêtre-catéchiste : quatre textes de hase, Montréal, Office Catéchistique Provincial, 1961.

39 Ibid.. pp. 28-54.

40 Pierre R.NWEZ, op. cit., p. 750. 
Il s'ensuit donc que la catéchèse franchit une nouvelle étape de son histoire. Non seulement elle dépasse une conception du contenu doctrinal qui ressemble trop à un "code ${ }^{41}$, mais elle dépasse encore la recherche trop exclusivement pédagogique. C'est qu'en catéchèse se pose le problème concret de la Parole vivante de Dieu dans le temps. Ce problème rejoint la problématique d'une démarche anthropologique : un va-et-vient de la Parole de Dieu et de la parole de l'homme. L'effort catéchétique des années 60 consiste à se situer au cœur même de cette rencontre : à savoir comment, en évitant tout réductivisme théologique ou anthropologique. concevoir l'acte de catéchèse dans une dialectique d'incarnation.

\section{MENTALITÉS}

L'ensemble de l'étude historique précédente révèle assez bien la perspective individualiste, dualiste et intellectualiste de l'enseignement traditionnel et de l'apport nouveau de la catéchèse actuelle.

\section{LA DIMENSION INDIVIDUELLE}

La toute première catéchèse des missionnaires en terre québécoise véhiculait déjà une mentalité à tendance individualiste qui avait envahi l'enseignement religieux, la prédication et le catéchisme en France. Les luttes religieuses des derniers siècles avaient favorisé une certaine privatisation de la religion; elles avaient développé un certain individualisme qui depuis assez longtemps avait supplanté le dynamisme communautaire des premiers siècles. L'important était alors de "sauver son âme » et d' "aller au ciel». La conquête anglaise n'a fait qu'amplifier ce repliement sur soi et ce souci de défendre sa propre vie tant au plan matériel que spirituel.

Il ne faut pas se surprendre si la catéchèse prend des allures intellectualistes. Dès que l'enseignement s'est adressé aux enfants avec les apparitions des écoles sous leurs différentes formes, le catéchisme devint l'instrument par excellence pour transmettre les connaissances nécessaires au salut. À peu près tous les catéchismes canadiens ont véhiculé une foi fortement intellectualisée ${ }^{42}$.

Cependant, au début du $\mathrm{XX}^{\mathrm{c}}$ siècle, le développement des sciences humaines remet en question un tel enseignement religieux.

41 Réginald Marsol aIS, «D”hier à aujourd'hui. Trois décennies de catéchèse au Québec ", dans Le Soufflle, n" 49, octobre 1974, p. 70.

42 Étienne ForTIER, op. cit., t. 1, p. 7. 
D'abord c'est une recherche sur les méthodes actives et intuitives qui font l'objet de cette remise en question. Mais c'est particulièrement avec les dossiers de l'Office de Catéchèse du Québec que les orientations fondamentales de la catéchèse sont transformées : délaissant une perspective cosmogonique et cosmologique du monde, les rédacteurs des dossiers préfèrent une vision plus anthropologique du monde ${ }^{43}$.

Pour eux, la personne. qui est en devenir, est une, c'est-à-dire à la fois extériorité et intériorité. La corporéité est symbolique de l'être profond; l'existence est à la fois matérielle et spirituelle. L'homme est un JE. Lorsqu'il agit ct pense, il le fait dans la totalité de son être. Son originalité est spécifiée par sa capacité de faire des choix responsables et conscients en vue de son devenir plus. Capable de "nommer" les êtres, il peut les reconnaître comme autres et se reconnaître lui-méme comme différent ${ }^{44}$.

Par son activité et à travers les nécessaires ruptures que son existence implique, l'homme se saisit comme immanence et comme transcendance; il se perçoit comme une liberté individuelle qui constamment cherche à assumer la vie concrète et à réaliser ses aptitudes ${ }^{45}$.

C'est ainsi que, par ses choix, l'homme accueille les sens que contiennent déjà les réalités, pour les orienter dans la direction qu'il choisit. Par l'interaction de l'autorité des valeurs sur l'homme et de l'autorité de l'homme sur les valeurs, une nouvelle signification jaillit. L'ensemble de ces choix forme pour ainsi dire une idéologie signifiante de la vie.

43 Office Catéchistique Provincial, Un sens all roygge. Introdliction générale; document pour l'éducuteur, Montréal, Fides, 1968 ; OfFICE DE CATÉCHÈSE DU QUÉBEC, Un sens all voyage. Introduction à la pédagogic de Secondaire III : document de l'éducateur, Montréal, Fides, 1976.

44 Office Catéchistique: Provincial. Un séns all voyage, réflexion sur l'existence corporelle ; document pour l'éducateur, Montréal, Fides, 1968 ; OfFICE de CaTÉCHÈSE du QuÉBEC. Un sens all royage: existence corporelle; document de l'éducateur, Montréal, Fides, 1976.

45 OffFICE de CATÉCHÈsf DU QUÉBEC, $U$ n sens all voyage 2 : réflexion sur l'activité humaine: document pour l'éducateur. Montréal, Fides, 1968 ; OfFICE de CATÉCHÈse dU QuéBEC, Un sens all royage: activité humaine; document de l'éducateur, Montréal, Fides, 1976. 


\section{LAA DIMENSION COLLECTIVE}

Une deuxième observation concerne l'aspect sociologique, l'aspect communautaire en catéchèse. Quelques grandes insistances sociales ont, en effet, joué dans l'enseignement religieux tant en France qu'au Québec.

Avant le premier catéchisme canadien-français de $\mathrm{M}^{* \mathrm{r}}$ de SaintVallier, une tradition catéchétique est déjà établie en France. Au Moyen Âge, la catéchèse est surtout centrée autour de la paroisse. La prédication dominicale devient en fait le lieu par excellence de l'instruction religieuse. Elle s'adresse à des groupes d'adultes; elle compte sur les parents et les parrains-marraines pour parfaire l'éducation chrétienne des enfants. Durant cette période, la catéchèse est surtout marquée par l'environnement de la paroisse et de la famille et par l'ensemble de l'existence. Elle est donc socialement très riche.

Le retour aux valeurs de la culture antique avec l'avènement de la Renaissance apporte en éducation une ouverture sur les arts, sur la richesse des sciences naturelles et sur des méthodes plus adaptées aux élèves. En formation religieuse, la Réforme suscite un intérêt pour l'éducation de la foi des enfants à l'école. En réaction, l'Église catholique multiplie les catéchismes et les confréries de catéchistes en vue de l'éducation religieuse des adultes et des enfants. Il n'est pas exagéré de dire que cette période s'intéresse d'une façon particulière et systématique à l'éducation religieuse des enfants, éducation qui se fait surtout par l'intermédiaire des parents.

Au Québec, ce double mouvement de la formation religieuse des parents par la voie de la prédication dominicale et de la formation religieuse des enfants par la voie des parents et des écoles se maintient. Compte tenu des circonstances, des situations et des événements propres au Québec, la formation religieuse subsiste en NouvelleFrance grâce aux efforts des curés, des parents et des professeurs. Du début du régime français jusqu'à ces dernières décennies, chaque page d'histoire révèle cette dimension sociale en catéchèse : les curés de paroisse forment leurs ouailles par la prédication dominicale, la visitc des paroissiens et la promotion de l'enseignement religieux. Les mandements des évêques ne cessent de rappeler aux pasteurs, aux parents et aux maitres leur grave devoir d'éduquer les enfants dans la foi. Enfin, l'avènement progressif des écoles élémentaires et des écoles secondaires privées se fait de plus en plus remarquer. Cette collaboration entre l'Église et la famille assure le succès de l'Insti- 
tution catéchétique canadienne ${ }^{46}$. Durant ces siècles, l'enseignement religieux s'est maintenu, s'est adapté et s'est enrichi à partir d'un environnement à structure paroissiale et familiale ${ }^{47}$.

Les deux dernières guerres provoquent des transformations culturelles, sociales et économiques remarquables. L'effondrement des cadres et des sécurités traditionnelles apporte, surtout à partir de 1960, une nouvelle vision de la communauté et de la vie chrétienne et, par conséquent, de la catéchèse.

Avec l'avènement d'une pensée plus personnelle et plus engagéc dans l'histoire, la catéchèse, dans la foulée des mouvements d'action catholique, s'ouvre à la réalité sociale. Deux phases importantes semblent caractériser l'évolution et l'intégration de cette dimension sociale dans les dossiers catéchétiques actuels : un aspect psychosocial et un aspect plus collectif.

L'approche psycho-sociale insiste beaucoup sur le fait que l'enfant est un être qui est ouvert aux autres et a besoin des autres poür se réaliser. L'enfant, qui fait l'apprentissage de la confiance en lui-même, de nouveaux rôles et d'une certaine compétence, et l'adolescent, qui découvre progressivement son identité, son unicité et son originalité en confrontation avec les composantes du milieu, doivent s'intégrer à la société. Ils découvrent progressivement qu'ils sont des êtres relationnels et qu'ils se font par des relations.

En plus, l'homme est un être historique : il fait partic d'une société, il fait partie d'un cnsemble de citoyens plus ou moins organisés, il est concrètement lié à un passé et il engage l'avenir de la société dans laquelle il vit. Par son travail, l'homme découvre des formes de collaboration ct crée des relations réeiles en vue de son humanisation. Conscient de sa solidarité avec les autres et du devenir du vivre-ensemble, l'homme tente d'arrêter des choix, de les unifier et de les coordonner en un tout. Il se construit une idéologic: il donne sens à sa vie, il contribuc à l'amélioration du devenir collectif; bref, il s'insère dans l'histoire par sa participation aux valeurs socio-culturelles. Par ses choix, l'homme devient solidaire

46 Fernand Porter. o.f.m., op cit., pp. 261-272. Cf. M.D. Chenu, "Dimensions nouvelles de la chrétienté », dans Vie spirituelle, 53 (1937), pp. 332-334.

47 Pierre Ranw'ez, op. cit., p. 74. 
de ses pairs et de l'histoire collective ${ }^{48}$. Ainsi donc, par son activité scientifique et technique sur la matière, par son engagement dans les divers secteurs sociaux, économiques ou autres et par le jeu des influences d'ordre socio-culturel, l'homme contemporain prend conscience de la solidarité humaine, de son appartenance à la collectivité et de la dimension sociale de son être.

L'apport du renouveau catéchétique en ce domaine consiste surtout en un effort pour mettre en relief la dimension relationnelle de la personne humaine. Cette dimension sociale s'intègre à la personne même. Elle fait partie de son devenir, elle se situe à l'intérieur même du contenu catéchétique. La catéchèse renouvelée considère l'homme dans son historicité. Elle le voit comme quciqu'un qui dans des situations bien concrètes prend en charge son devenir avec ses pairs dans la visée de créer un milieu favorable à son humanisation, humanisation qui trouve sa plénitude dans la communauté ecclésiale.

\section{Perspective anthropologiQue}

L'histoire de la catéchèse manifeste également le passage d'une catéchèse anthropocentrique à une catéchèse anthropologique.

Dans la Renaissance, en effet, plusieurs pédagogues se refusent à livrer un enseignement livresque. Partant d'une meilleure connaissance de l'homme, ces éducateurs essaient d'adapter l'enscignement à l'enfant afin de le rendre capable de s"intégrer dans la société. Suite au Concile de Trente, les protestants et les catholiques rivalisent en catéchètes et en catéchismes qui s'adressent aux enfants. Sous le régime français, le catéchisme québécois, malgré ses faiblesses pédagogiques, vise dans son ensemble beaucoup plus l'éducation que l'instruction de l'enfant. Même si une grande importance est attribuée à la connaissance des «vérités » religicuses, la pratique religieuse est encore très prépondérante. Tous s'efforcent d'améliorer la méthode des catéchismes afin de mieux adapter le contenu aux différents âges et d'en faciliter la pratique ${ }^{49}$. Done les corrections se concentrent sur le contenu, le langage, la méthode et cherchent à répondre à la maturation psychologique des enfants.

48 Offict: Catíchistique. Provincial, La force des rencontres: homme

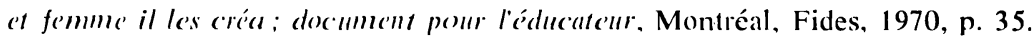

49 M"r Olivier BrinNI), "Mandement de M*r l'lllustrissime et Révérendissime évêque de Québec au sujet du catéchisme », dans $M E Q$, II, p. 288. 
À cette préoccupation constante de l'enfant en enseignement religieux, les évêques accordent une importance capitale à l'environnement. Ce rôle de l'environnement qui caractérise particulièrement le Moyen Âge n'a jamais cessé de s'exercer au cours de toute l'histoire de la catéchèse, du moins au Québec. La ténacité des catéchistes, le rôle des curés et des prêtres, l'importance de la pratique dominicale, le témoignage constant de chaque membre de la famille contribuent à faire naître, à développer et à appuyer l'enfant dans son cheminement religieux. C'est toute l'institution catéchistique qui montre jusqu'à quel point le milieu a joué dans l'enseignement religieux. Cet environnement s'affaiblira progressivement avec la montée de la scolarisation. Pour des raisons culturelles, éducatives et pédagogiques, l'accent en enseignement religieux se déplace de l'environnement à l'école ${ }^{50}$.

Ainsi donc, ce souci constant des éducateurs de la foi pour l'enfant, cette présence assidue d'un environnement religieux et cette intrusion des méthodes actives et intuitives en catéchèse démontrent suffisamment que cette diniension. anthropologique. sous une certaine forme, n'est pas de date récente en catéchèse ${ }^{51}$. Mais cette perspective anthropologique, fondée sur la nature abstraite de l'homme, revêtait la pratique catéchistique dune couleur anthropocentrique ${ }^{52}$.

La catéchèse nouvelle veut remédier à cette conception plutôt figée de l'homme. Elle propose une conception de l'anthropologie de type philosophique.

La catéchèse ancienne rendait difficile la relation entre la foi et l'existence surtout en raison de sa philosophie de type dualiste. Le renouvellement part de l'honme lui-même. C'est alors dépasser la simple adaptation pédagogique pour resituer, dans son ensemble. l'Église dans ses rapports avec le monde. Le développement récent des sciences humaines et l'avènement d'une théologie de la sécularisation font que la catéchèse porte une oreille plus attentive à l'anthropologie prise dans son sens philosophique. c'est-à-dire dans

50 Fr. X. Ross, L'enseignement religieux dans la famille. à l'école, alu collège, à l'Université et dans la vice chrétienne, Montréal. École sociale populaire. 1930 (École sociale populaire, 196), p. 178.

51 André POLAerT, "Anthropologie et vie de foi dans la Catéchèse ». dans Catéchès's. t. 7, n" 27, avril 1967, p. 158.

52 René Brochu. "À propos de catéchèse", dans Églisc canadicnne. v. $5, \mathrm{n}^{\prime \prime}+$, avril 1972 , p. 105 . 
le sens d'une réflexion humaine qui tente de dégager une vision de l'homme et de sa relation à la nature, un sens de l'existence et un sens de l'histoire humaine ${ }^{53}$.

Cet homme, dans la perspective catéchétique, devient médiation de langage. L'homme est «l'alphabet de Dieu ${ }^{54}$ ». À travers les expériences humaines, l'immanence de Dieu se laisse découvrir ${ }^{55}$. L'expérience humaine éclaire la Parole de Dieu et la Parole de Dieu éclaire l'expérience humaine. Il faut noter qu'avec un enseignement religieux multidimensionnel ${ }^{56}$, l'expérience humaine comprend le vécu du jeune et de la foi en Jésus, le vécu collectif du monde et celui des chrétiens.

Le renouveau catéchétique apporte donc un changement radical dans l'éducation de la foi. Non seulement cette catéchèse se fait plus adaptée à l'adolescent avec l'usage des méthodes actives et intuitives mais elle considère l'étudiant comme «lieu » de la catéchèse. Grâce à une pensée plus existentialiste et personnaliste, la catéchèse découvre avec un regard neuf la présence de Dieu dans la condition humaine, ou plutôt elle découvre progressivement en l'homme Jésus la vocation divine de l'homme qui éclate dans le mystère pascal. La catéchèse actuelle met en relief ce rapport intime et cet éclairage mutuel de l'expérience humaine et de la Parole de Dieu. En effet. dans le langage biblique, la Parole de Dieu s'exprime toujours par la médiation de la condition humaine qui atteint sa plénitude d'expression en l'homme Jésus. Par ailleurs, c'est constamment à partir de sa condition humaine que l'homme peut saisir Dieu. Enfin, la découverte de l'Église comme communauté et comme peuple de Dieu, comme signe de salut et d'unité marque un troisième apport de cette catéchèse renouvelée.

Ce renouveau catéchétique semble permettre à l'étudiant de se mieux saisir lui-même, de mieux appréhender la personne du Christ

53 Rohert COFFY, "Théologie et Anthropologie ». dans Catéchèse. t. 8, n" 32, juillet 1968, p. 321 : cf. Office Catéchistique: Provincial. Un se'ns au voyage. Introduction générale; document pour l'éducaterur, Montréal, Fides, 1968 , p. 6.

54 Jean LEDu, «Catéchèse et anthropologie », dans Catéchèse, t. 6 , n" 24, juillet 1966, p. 296.

55 André Brien, "Anthropologie et Catéchèse», dans Catéchistes. t. 25, n"100, octobre 1974, p. 613.

56 OfFICE DE CATÉCHÈSE du QuÉBEC. Un sens all royage. Introduction ¿̀ la pédagogie de Secondaire III: document de l'éducateur, Montréal, Fides. 1976, p. 4. 
et de s'engager davantage dans la communauté chrétienne selon l'appréciation de nombreux pasteurs, catéchètes, parents et étudiants. Cette catéchèse a sans doute permis à plusieurs durant ces deux dernières décennies de mieux maintenir une foi remise en question, de la purifier et de la rendre plus adulte.

Mais il est encore trop tôt pour dresser un bilan ou mesurer les effets réels et durables de cette toute récente réforme de linstitution catéchétique québécoise. Aux historiens et aux générations à venir d'en juger.

Raymond ANCTIL,

Université du Québec, Trois-Rivières. 